\title{
High-resolution design of a protein loop
}

\author{
Xiaozhen Hu, Huanchen Wang, Hengming Ke, and Brian Kuhlman* \\ Department of Biochemistry and Biophysics, University of North Carolina, Chapel Hill, NC 27599 \\ Edited by David Baker, University of Washington, Seattle, WA, and approved September 24, 2007 (received for review August 23, 2007)
}

Despite having irregular structure, protein loops often adopt specific conformations that are critical to protein function. Most studies in de novo protein design have focused on creating proteins with regular elements of secondary structure connected by very short loops or turns. To design longer protein loops that adopt specific conformations, we have developed a protocol within the Rosetta molecular modeling program that iterates between optimizing the sequence and conformation of a loop in search of low-energy sequence-structure pairs. We have tested the procedure by designing 10-residue loops for the connection between the second and third strand in the $\boldsymbol{\beta}$-sandwich protein tenascin. Three low-energy designs from 7,200 flexible backbone trajectories were selected for experimental characterization. All three designs, called LoopA, LoopB, and LoopC, adopt stable folded structures. Highresolution crystal structures of LoopA and LoopB have been solved. LoopB adopts a structure very similar to the design model $(0.46 \AA$ rmsd), and all but one of the side chains are modeled in the correct rotamers. LoopA crystallized at low $\mathrm{pH}$ in a structure that differs dramatically from our design model. It forms a strand-swapped dimer mediated by hydrogen bonds to protonated glutamic acids. Gel filtration indicates that the protein is not a dimer at neutral pH. These results suggest that the high-resolution design of protein loops is possible; however, they also highlight how small changes in protein energetics can dramatically perturb the low free energy structure of a protein.

computational protein design | loop modeling | tenascin

D rote rotein loops often adopt specific conformations that are critical to protein function. Many protein active sites contain residues that are located in loops, and protein-protein interactions are frequently mediated by loops. Despite the clear importance of loops, most studies in de novo protein design have not involved the creation of longer loops that adopt specific conformations but rather have focused on proteins that consist almost entirely of $\alpha$-helices or $\beta$-strands connected by short turns or loops (1-8).

There are several reasons why designing ordered loops may be especially challenging. Unlike $\alpha$-helices and $\beta$-sheets, the backbone hydrogen bonding potential of a loop is not automatically satisfied. For a loop conformation to have low free energy, it is important that each polar group in the loop is hydrogen bonding with another group in the protein or is accessible to water. However, if too much of the loop is exposed to water, then it is less likely that it will adopt a unique conformation. Additionally, unlike $\alpha$-helices and $\beta$-strands, there are not well determined amino acid preferences for forming a well ordered loop. Protein engineers have shown that helices can be built by favoring sequences rich in alanine, leucine, lysine, and glutamate, whereas the $\beta$-branched amino acids, threonine, valine, and isoleucine, prefer to form $\beta$-strands (9-11). Loops can be designed by favoring sequences enriched in glycine and polar amino acids, but sequences of this type are unlikely to form a unique conformation (12). Well ordered loops typically have diverse sequences that form specific tight packing interactions within the loop and with the rest of the protein.

Designing a new loop requires specification of the new backbone coordinates and the amino acid sequence. This is a difficult problem, because most arbitrarily chosen protein backbones are unlikely to be designable, i.e., there will be no amino acid sequences that pack on the structure with energies that are comparable with what are observed for naturally occurring proteins (13). Designable backbones can be created by using heuristics derived from naturally occurring protein structures, or structure prediction protocols can be used in tandem with sequence optimization protocols to search for low-energy sequence-structure pairs $(2,6,14-18)$. Here, we examine whether the second approach, combining structure prediction with automated sequence optimization, can be used to design loop sequences that adopt unique conformations. Schliebs and coworkers (19) have used a similar strategy to introduce four mutations into an eight-residue loop in triosephosphate isomerase. In their study, Monte Carlo sampling of loop conformations was interspersed with hand-picked mutations.

A variety of methods have been developed to predict the structures of protein loops (20). In general, loop prediction protocols have two primary components, a procedure for searching through the various conformations a loop might adopt and an energy function for evaluating the relative favorability of these conformations. Often, conformational sampling is aided by using loop conformations from other proteins as starting points for structure optimization. We use the molecular modeling program Rosetta to perform sequence design and loop modeling. Rosetta was first developed for $a b$ initio structure prediction but has since been expanded to contain protocols for high-resolution structure refinement, loop modeling, molecular docking, and protein design $(6,21-24)$. The Rosetta energy function used for high-resolution refinement and design emphasizes short range interactions: steric repulsion, van der Waals interactions within $5.5 \AA$, torsion energies, hydrogen bonding, and a desolvation penalty for bringing atoms close to other polar atoms $(25,26)$. Sequence and conformational space are searched with a Monte Carlo optimization procedure. Single amino acid substitutions or backbone torsion angle perturbations are evaluated with the Metropolis criterion. In the last stage of refinement, the Monte Carlo moves are followed by gradientbased minimization of torsion angles before comparing the energy of the structure to the most recently accepted structure. To design protein loops, we have combined Rosetta's loop modeling protocols with sequence optimization. The protocol iterates between refining the structure of a loop and designing a sequence for the loop. We generally perform thousands of independent trajectories, because each individual simulation eventually gets trapped in a local energy minimum.

To experimentally test the protocol, we have examined whether we can design new backbone conformations and sequences for the 10-residue loop that connects the second and third $\beta$-strands from the third fibronectin type III domain from tenascin-C (27). In these studies, the WT loop is removed from the protein, and the new loop

Author contributions: X.H. and B.K. designed research; X.H., H.W., and H.K. performed research; H.W. and H.K. contributed new reagents/analytic tools; X.H., H.W., H.K., and B.K. analyzed data; and X.H. and B.K. wrote the paper.

The authors declare no conflict of interest.

This article is a PNAS Direct Submission.

Abbreviation: SASA, solvent accessible surface area.

Data deposition: $X$-ray coordinates and structure factors have been deposited in the Protein Data Bank, www.pdb.org [PDB ID codes 2RB8 (LoopB) and 2RBL (LoopA)].

*To whom correspondence should be addressed. E-mail: bkuhlman@email.unc.edu.

This article contains supporting information online at www.pnas.org/cgi/content/full/ 0707977104/DC1.

() 2007 by The National Academy of Sciences of the USA 
is designed from scratch. The naturally occurring loop that connects these strands forms a well ordered structure with low B-values in the crystal structure, and ${ }^{15} \mathrm{~N}$ nuclear spin relaxation experiments have shown that the loop is fairly rigid in solution (28). Insertion of four glycines into the same loop from the homologous protein $\mathrm{FNfn} 10$ lowers the stability of FNfn10 by $1.7 \mathrm{kcal} / \mathrm{mol}(1 \mathrm{cal}=4.18 \mathrm{~J})(29)$.

\section{Results and Discussion}

Computational Design. New backbone conformations and sequences were designed for residues 22-31 of the third fibronectin type III domain from tenascin-C (27). A three-step process was used to create the new loops. First, starting backbone conformations for loop design were picked from fragments of naturally occurring proteins. Second, iterative rounds of backbone optimization and sequence design were used to search for low-energy sequencestructure pairs. Third, a variety of calculated energies were used to pick designs for experimental validation.

Starting loop structures were built by searching the Protein Data Bank (PDB) database for 12 residue fragments of naturally occurring proteins with endpoints that superimpose with low rmsd on the backbone atoms of residues 21 and 32 of tenascin. One hundred forty-two fragments were identified with endpoint rmsd values of $<3 \AA$. These fragments were then grafted onto tenascin, using Monte Carlo optimization and gradient-based minimization of backbone torsion angles with a scoring function that favored loop closure, low-energy backbone torsion angles, low-energy backbonebackbone hydrogen bonds, and the absence of clashes with neighboring backbone atoms. From these simulations, 36 low-scoring backbone structures were selected for use as starting points for high-resolution design. These loops varied in rmsd to the WT loop from 0.7 to $2.5 \AA$ [supporting information (SI) Fig. 7].

Each starting structure was used to seed 200 independent sequence design and backbone optimization trajectories (7,200 trajectories in total). Each trajectory consisted of nine rounds of sequence optimization followed by torsion-based backbone and side-chain optimization (see Materials and Methods). During these runs, only small backbone perturbations were made, and the average backbone perturbation over the course of a trajectory was $0.3 \AA$ rmsd. However, these small backbone perturbations often changed the most preferred sequence; out of the 10 loop residues, it was common to see four or five mutations when comparing with the initial designed sequence, and the Rosetta full atom energy of the designed structure typically dropped between 5 and $10 \mathrm{kcal} / \mathrm{mol}$ (Fig. 1 and Table 1). In some cases, the simulation fell into a local minimum after the first round of design and backbone optimization, whereas, in other cases, the energy continued to drop throughout the trajectory. For the 7,200 design models, the Rosetta scores varied from -135 to $-156 \mathrm{kcal} / \mathrm{mol}$. Some starting structures produced lower-energy high-resolution models on average than other starting structures. Ten starting structures were represented in the final top 200 scoring models.

Selecting Designs for Experimental Validation. Designs were selected for experimental validation, using several criteria: the Rosetta full atom score, the number of buried polar groups without a hydrogen bonding partner (30), and the quality of packing in the protein as measured by a score that evaluates the amount of molecular surface accessible to a $0.5 \AA$ radii probe but not a water molecule [solvent accessible surface area (SASA)pack score; see Materials and Meth$o d s$ ] (31). A first round of selection was made by eliminating all models with more than two unsatisfied hydrogen bonds in the region of the designed loop, an average SASApack score per residue of $>2.0 \AA^{2}(90 \%$ of protein crystal structures in the protein database have average per-residue SASApack scores $<2.0 \AA^{2}$ ) and a total score of $>-148 \mathrm{kcal} / \mathrm{mol}$. For reference, WT tenascin has a Rosetta score of $-148 \mathrm{kcal} / \mathrm{mol}$ after relaxation, two unsatisfied hydrogen bonds in the region of the designed loop, and an average SASApack score per residue of $0.83 \AA^{2}$. The hydrogen bond filter
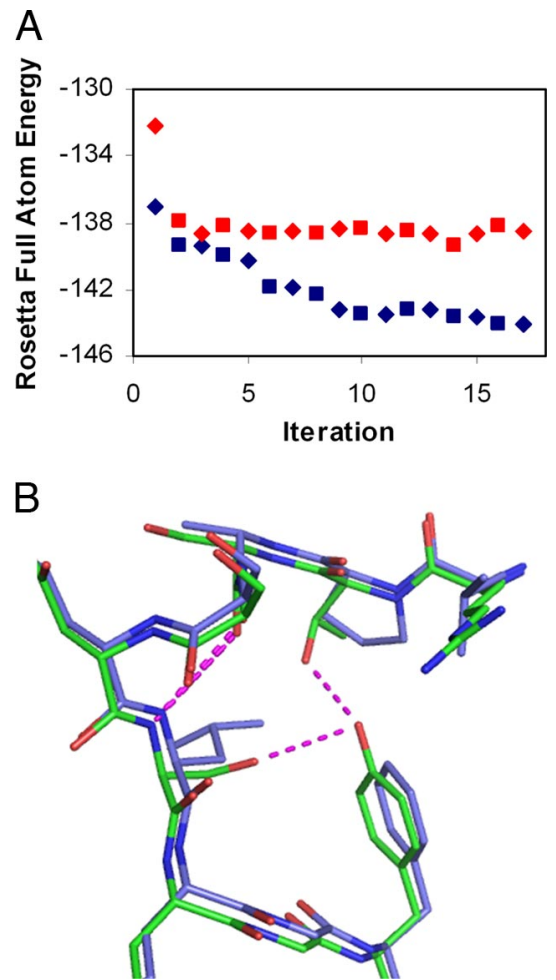

Fig. 1. Iterative optimization of a loop sequence and conformation. (A) Two representative design trajectories are shown in red and blue. The diamonds indicate the energy of the protein after sequence design, and the squares indicate the energy of the protein after optimization of backbone and sidechain torsion angles. $(B)$ The starting (green) and ending (blue) models for the red trajectory shown in $A$.

was the most stringent and removed 6,608 models from consideration. Sixty models were left after applying all three filters. From these 60, three sequences were selected for further study: the lowest-scoring structure (LoopC) and two structures with low packing scores (LoopA and LoopB) (Fig. 2 and Table 2). Each sequence differs from the WT protein in at least 7 of the 10 sequence positions. The designed sequences also differ from the protein loop fragments that were used in the first stages of the design process in at least 7 of the 10 sequence positions.

The WT loop on tenascin is stabilized by a set of hydrophobic residues that form closely packed interactions in the space between the beginning and end of the loop. Similar types of interactions are present in all three designs selected for experimental study, but the identities of the amino acids vary (Fig. 2). The pairwise backbone rmsd values between the designs range between 0.9 and $1.6 \AA$ (SI Table 3). To evaluate whether the designed sequences were specific for their respective backbones, the sequences were threaded onto the other design models and scored after rotamer repacking, and every sequence favored the backbone for which it was designed (SI Table 4). For example, LoopA has a score of $-152 \mathrm{kcal} / \mathrm{mol}$; when the LoopA sequence is threaded onto the WT, LoopB, and

Table 1. The starting and ending sequences scored on the starting and ending structures from the red trajectory shown in Fig. $1 A$

\begin{tabular}{lcc} 
& \multicolumn{2}{c}{ Sequence } \\
\cline { 2 - 3 } Structure & Starting & Ending \\
\hline Starting & -133.1 & -131.3 \\
Ending & -138.5 & -139.4 \\
\hline
\end{tabular}



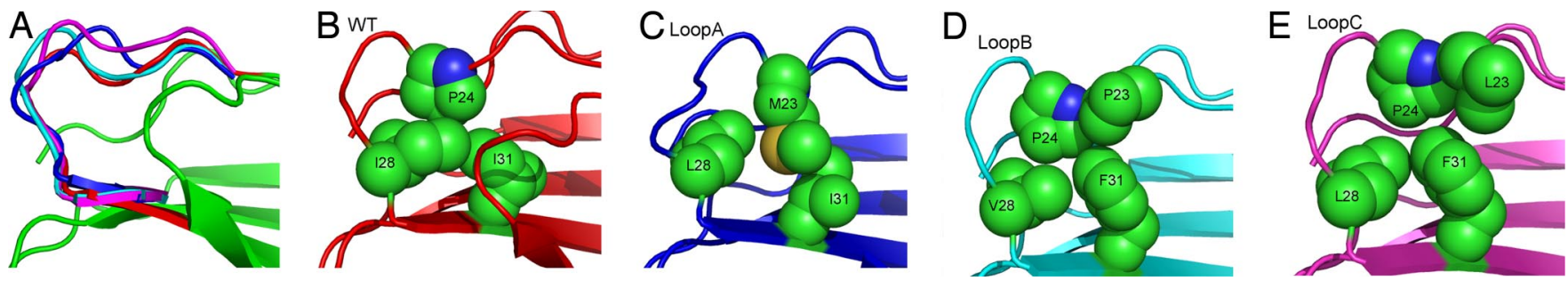

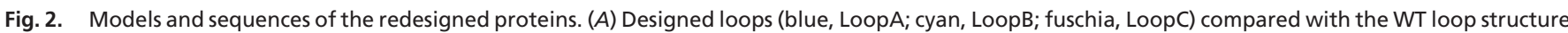
(red, WT). (B-E) Models of the WT and the designed loops.

LoopC backbones the scores are $-137,-140$, and $-143 \mathrm{kcal} / \mathrm{mol}$ respectively.

Structure Prediction with the Designed Loop Sequences. During the loop design protocol, small perturbations in backbone motion are used to look for local minima in structure space, but the protocol does not include any explicit tests that would probe whether the design sequence prefers significantly different alternative conformations. To computationally test whether our designed sequences prefer the designed target conformations, we performed structure prediction with the loop sequences. In these simulations, the only input into Rosetta is the sequence of the loop and the structure of the scaffold. Structure prediction is performed by using fragmentbased insertion with a round of low-resolution scoring followed by high-resolution scoring (32). A cyclic coordinate descent algorithm is used to close loops after insertions (33). As is the usual strategy with Rosetta, thousands of independent structure prediction simulations were run with each sequence. For all three sequences, the lowest-energy structure predictions resembled the design models (backbone rmsd $<0.8 \AA$ ), and there was an increase in energy as the rmsd values for the models increased by $>1 \AA$ (Fig. 3). Pairwise backbone rmsd values were calculated between each of the designed loops and the lowest-energy structure prediction for each loop (SI Table 4). There was a closer agreement between matched pairs, i.e., LoopA structure prediction compared with the LoopA design, than there was between unmatched pairs, i.e., LoopA structure prediction compared with the LoopB design model.

The lowest-energy structure predicted for LoopB is similar to the design model (backbone rmsd $=0.77 \AA$ ), but there are noticeable differences in the backbone positions of residues $24-26$. In the predicted structure, the carbon alpha positions of these residues are shifted $\approx 1 \AA$ toward the N-terminal tail of tenascin, and proline 24 is better packed in the structure prediction model. The SASAprob score (see Materials and Methods) of proline 24 is 0.29 in the structure prediction model and 0.09 in the original design model. During structure prediction with the LoopB sequence, there were models created that more closely matched the LoopB design model (backbone rmsd $<0.3 \AA$ ), but these models scored worse (Fig. 3).

Table 2. Sequences and scores of the redesigns

\begin{tabular}{lccccc} 
& & \multicolumn{3}{c}{ SASAprob } \\
\cline { 3 - 5 } & & \multicolumn{3}{c}{ Residue } & \\
\cline { 3 - 5 } Protein & Total Score & 24 & 28 & 31 & Sequence \\
\hline WT & -148 & 0.42 & 0.05 & 0.05 & $22-$ FKPLAEIDGI-31 \\
LoopA & -152 & 0.12 & 0.52 & 0.52 & SMQLSQLEGI \\
LoopB & -149 & 0.09 & 0.09 & 0.09 & MPPSQPVDGF \\
LoopC & -156 & 0.002 & 0.02 & 0.02 & ALPSRPLDGF
\end{tabular}

The SASAprob scores, which reflect the quality of packing for individual residues, are shown for residues buried in the center of the loop. A SASAprob score of 0.20 for a leucine indicates that $80 \%$ of the leucines in a similar environment in the PDB are better packed (see Methods).
Our design procedure did not find the lower-energy conformations for the LoopB sequence, indicating that in the future that it will be advantageous to perform more aggressive sampling of conformational space, including de novo structure prediction, when iterating between sequence design and structure optimization.

Experimental Characterization. All three designed proteins were expressed in Escherichia coli. One-dimensional ${ }^{1} \mathrm{H}$ NMR spectra of each protein indicate that they are well folded and adopt $\beta$-sheet structures (SI Fig. 8). Circular dichroism was used to probe the thermal stability of the proteins (Fig. 4). LoopB and LoopC have midpoints of thermal unfolding $\left(T_{\mathrm{m}}\right)$ that are similar to the WT protein, whereas the $T_{\mathrm{m}}$ for LoopA is $15^{\circ} \mathrm{C}$ lower than the that of WT protein. All three proteins return to the folded state when the temperature returns to $25^{\circ} \mathrm{C}$.

Crystal trays were set up for all three designs. Crystals that diffract at high resolution were obtained for LoopA and LoopB. The crystal structure for LoopB was solved to a resolution of 1.45 $\AA$ with an $R$ factor of $17 \%$ and an $R_{\text {free }}$ of $19 \%$. There is good agreement between the design model and the crystal structure. The design model was superimposed on the crystal structure by aligning the loop residues and residues that make contact with the loop (residues 4-8, 20-31, 48-55, and 72-74) (Fig. 5). Based on this superposition, the rmsd between the backbone atoms in the designed loop and the crystal structure was $0.46 \AA$. The lowest-energy de novo structure prediction for LoopB had a slightly better match to the crystal structure, the rmsd was $0.42 \AA$. In the crystal structure the backbone atoms of residues 24-26 were located between the design model prediction and the de novo prediction. The $\mathrm{B}$ values for the atoms in the redesigned loop are comparable with the other loops in the protein, and are all $<30 \AA^{2}$.

All of the side-chain rotamers in the design model were predicted correctly except for GIn-26. In the crystal structure, the side chain of Gln-26 forms hydrogen bonds with the backbone nitrogen and side-chain oxygen of Asp-2 (Fig. 5) and has low B values $(<25)$. We were curious whether this side chain was not predicted correctly because of changes in the backbone coordinates of the residues or because of the Rosetta energy function. To test between these two options, the backbone coordinates of the crystal structure were used as the template for a Rosetta side-chain repacking simulation with the designed sequence. Like the design model, the repacked structure did not have hydrogen bonds between Asp-2 and Gln-26. The Rosetta energy function disfavors the crystal structure rotamer for several reasons. First, the rotamer adopted in the crystal structure is especially rare in the Dunbrack rotamer library $(34,35)$, and Rosetta assigns an internal energy of $3.0 \mathrm{kcal} / \mathrm{mol}$ to this rotamer. The Rosetta-preferred rotamer has a rotamer score of 0.8 $\mathrm{kcal} / \mathrm{mol}$. Second, Rosetta only assigns weak scores to the two putative hydrogen bonds. Both have scores weaker than $0.5 \mathrm{kcal} /$ mol because the distances and angles between the groups are suboptimal. Favorable hydrogen bonds in Rosetta score near 2 $\mathrm{kcal} / \mathrm{mol}$. Third, there is a desolvation penalty $(>3 \mathrm{kcal} / \mathrm{mol})$ for removing the glutamine side chain from water. It is difficult to determine which of these scores is most misrepresenting the true 

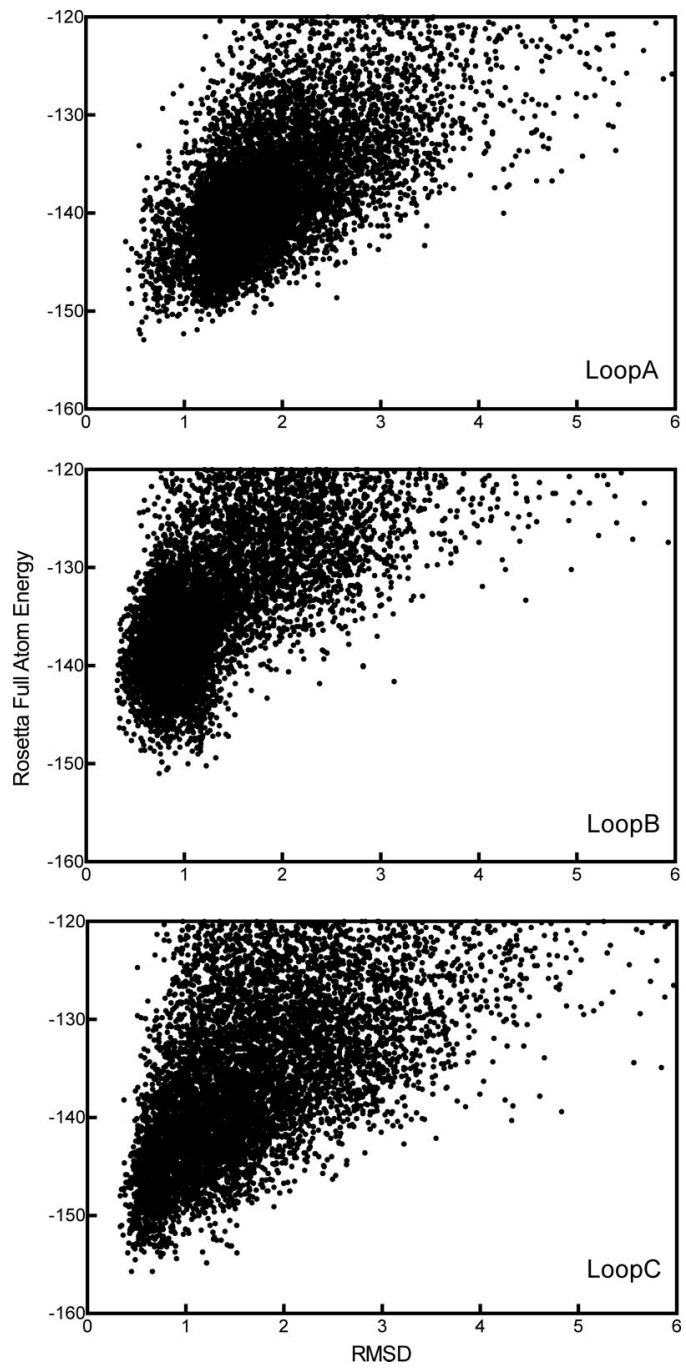

Fig. 3. Structure prediction with the designed sequences. Ten thousand independent prediction trajectories were run for each design, and the Rosetta energies of the models were plotted against the backbone rmsd of the predicted structures compared with the design models.

energetics of Gln-26, and this result demonstrates the challenge of balancing nearly equal and opposite energy terms.

LoopA crystallized at low $\mathrm{pH}$ (3.0). The resolution of the structure was $2.1 \AA$ with an $R_{\text {free }}$ value of $30 \%$. Under these conditions, the structure of the protein does not look similar to the design model. In the crystal, the protein adopts a 50/50 mixture of domain-swapped dimers and monomers (Fig. 6). In the domainswapped structure, the designed loop opens up to allow strands 1 and 2 to insert into the partner molecule. The loop is stabilized by a set of hydrogen bonds involving acidic side chains that appear to be protonated. Unfortunately, there is not clear electron density for the loop residues in the monomeric chain. This suggests that, at low $\mathrm{pH}$, the loop is not adopting a specific conformation in the monomer. To further characterize the monomer-dimer equilibrium, gel filtration experiments were performed at a variety of $\mathrm{pHs}$. At neutral $\mathrm{pH}$, the protein has an apparent molecular mass that is close to the predicted mass for a monomer, whereas at low $\mathrm{pH}$, the apparent molecular mass is $18 \mathrm{kDa}$, halfway between a monomer and dimer. In both cases, the concentration of the protein sample loaded on to the gel filtration column was $2 \mathrm{mM}$. These results, combined with the decreased stability of LoopA at pH 7, suggest that the designed loop may not have a strong preference for adopting the target conformation.

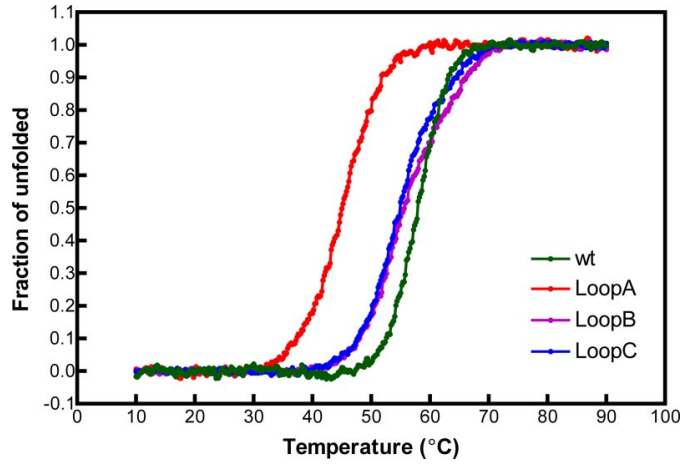

Fig. 4. Thermal unfolding of the designed sequences as monitored with circular dichroism.

None of the scores that were used to evaluate the design models suggested that LoopA would be more prone than the other loop designs to forming a domain swap interaction. Domain swapping has been observed in many proteins, including de novo designed proteins $(36,37)$. From these studies, it is clear that subtle changes in environment or sequence can promote swapping. Unlike the sequences of LoopB, LoopC, and the WT protein, LoopA does not have any prolines in the redesigned loop. In the domain-swapped crystal structure of LoopA, residues 23, 27, 29, and 30 have $\phi$ angles that are incompatible with a proline. This suggests that the prolines in the other designed loops could play a role in preventing domainswapping by disallowing conformational changes required for opening up the loop. However, it should be noted that, in a previous
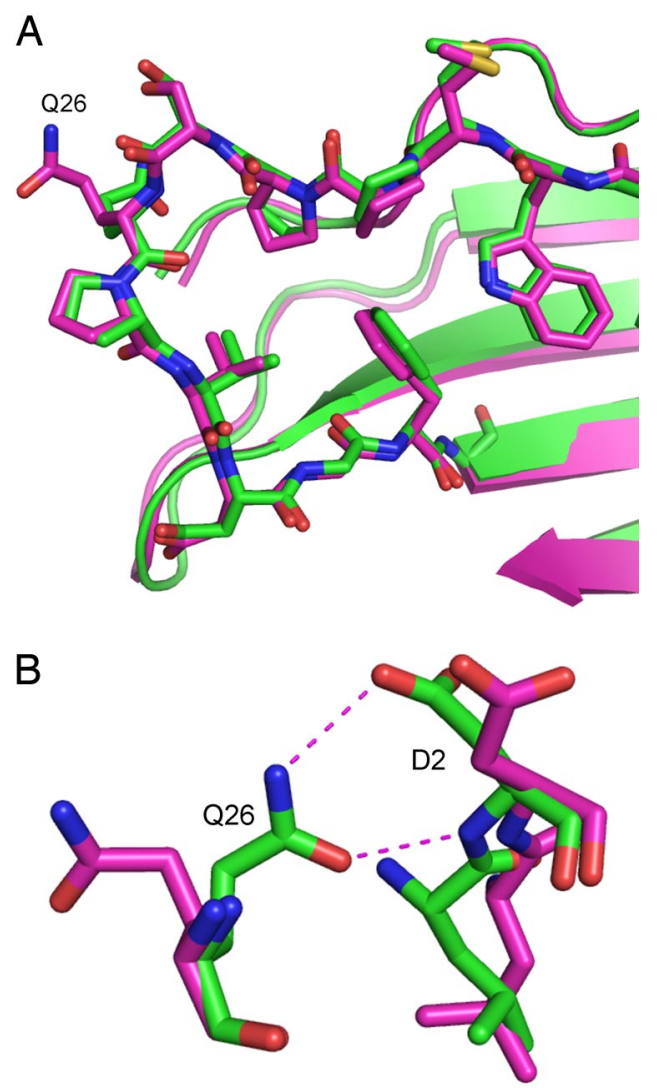

Fig. 5. Structural alignment between the crystal structure and the design model. $(A)$ The crystal structure of LoopB (green) aligned with the design model of LoopB (mauve). The backbone atoms of residues $4-8,20-31,48-55$, and 72-74 were used for the alignment. $(B)$ Close-up of glutamine 26. 
A

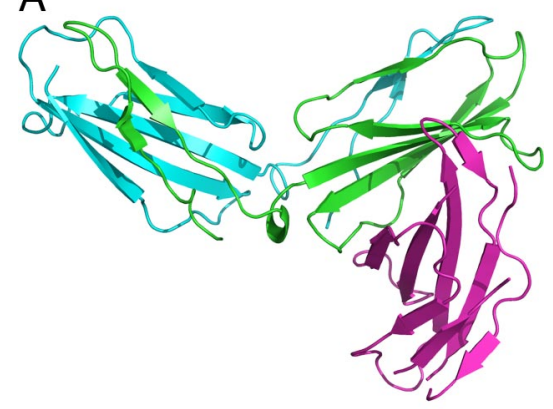

B

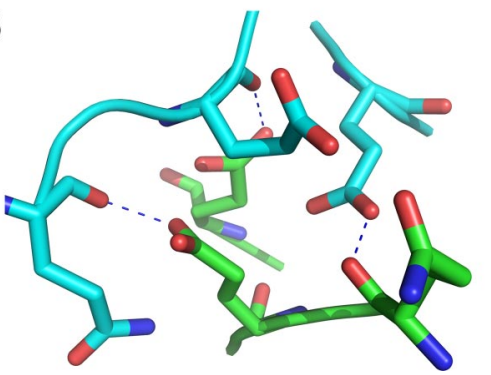

Fig. 6. The crystal structure of LoopA at low pH. $(A)$ The repeating unit contains a domain-swapped dimer (cyan, chain 1; green, chain 2) and a monomer (purple). Electron density is not present for the redesigned loop in the monomer. In the dimer, the loop opens up, and strands 1 and 2 insert into the partner molecule. $(B)$ The designed loop appears to be stabilized by protonated glutamic acid residues.

study, we used a designed proline to favor domain swapping (38). In summary, the LoopA results highlight the diversity of structures that a designed sequence can adopt and shows that explicit negative design would be useful if the competing states could be identified a priori.

\section{Conclusion}

Our results indicate that, with the current Rosetta energy function and sampling techniques, it is possible to design a 10-residue loop with high accuracy. LoopB is stabilized by tight packing interactions between hydrophobic side chains in the center of the loop. In the future, it will be interesting and important to test whether novel protein loops can be designed by forming new hydrogen bonding interactions. In this study, we used de novo structure prediction simulations to test whether our designed sequences prefer the target conformations. In all three cases, the lowest-energy structure prediction resembled the design models, but, in the case of LoopB, there were a few residues that were shifted by $\approx 1 \AA$ from the design model. In the crystal structure of LoopB, these residues adopted a position that was between the design model and the structure prediction. In future designs with more complicated target structures, it may be even more useful to evaluate designed sequences with de novo structure prediction simulations. These simulations can provide templates for creating even lower-energy sequencestructure pairs, and they can be used to determine whether negative design will be needed to disfavor competing states.

\section{Materials and Methods}

Iterative Backbone and Sequence Optimization. Rosetta's standard full atom energy function was used for structure prediction and sequence design $(6,21)$. Rotamer-based sequence optimization was performed as described in ref. 39. Dunbrack's backbone-dependent rotamer library was used with extra subrotamers created by varying all $\chi 1$ angles and the $\chi 2$ angles on aromatic residues plus or minus one standard deviation from the most preferred $\chi$ angles (34). Rosetta's backbone torsion angle energies are based on the prob- ability of observing a particular $\phi$ and $\psi$ angle for a given amino acid in the protein database (21). During sequence design, all 10 residues in the designed loop were allowed to vary to any amino acid except for cysteine, and the neighboring residues were allowed to adopt alternative side-chain conformations. Nine rounds of iterative sequence design and backbone optimization were used to search for low-energy sequence-structure pairs.

Backbone flexibility was restricted to the loop. Backbone optimization was performed by using Monte Carlo optimization. Only torsion angles are explicitly varied during the procedure. A single Monte Carlo move consisted of ( $i$ ) a small change to the $\phi$ and $\psi$ angles of the loop residues (up to five residues are varied simultaneously), (ii) a quick optimization of side-chain rotamers, and (iii) gradient-based optimization of backbone and side-chain torsion angles. After performing these three steps, the energy of the new structure is compared with the energy of the protein before the move, and the Metropolis criterion is used to decide whether the move should be accepted. Two types of moves were used to create the initial perturbation to the backbone: small random changes $\left(\approx 1^{\circ}\right.$ perturbations $)$ and shear moves. A shear move consists of a small change to a $\phi$ angle compensated by a change in the opposite direction to the $\psi$ angle. Fast rotamer optimization was performed by cycling over each side chain once (in random order) and choosing the lowest-energy rotamer given the current environment. Gradient-based minimization was performed with a conjugate gradient protocol that calculates the first derivative of the energy function for each torsion angle that is being varied $(21,22)$. A score that favors a low rmsd between the first and last residue of the loop and the protein scaffold was used to keep the loop closed during backbone optimization.

De Novo Structure Prediction of Loop Sequences. The structures of the designed sequences were predicted by using a recently developed Monte Carlo-based loop modeling protocol in Rosetta (40). The sequence of the loop is used to pick overlapping three-residue fragments from the PDB with similar sequences. These fragments are then randomly combined to create a starting structure for optimization. The first round of optimization is performed with a low-resolution model of the protein that favors good backbone torsion angles and backbone hydrogen bonding. The second round is performed in high-resolution full atom mode and combines small and shear moves (see above) with gradient-based minimization and Dunbrack's cyclic coordinate descent algorithm for loop closure (33). Rotamer repacking is also performed after every 20 backbone trials. Thousands of Monte Carlo moves are considered. For each sequence, 10,000 independent trajectories were performed.

SASApack and SASAprob Scores. A score based on accessible surface areas (SASApack score) was used to evaluate the quality of packing of the design models. The SASA of each residue in the protein was calculated with two different probes (radii equal to $0.5 \AA$ and $1.4 \AA$ ), and the difference was compared with the average difference seen in the PDB for a particular amino acid in a similar buried environment (31). A larger-than-average difference between SASA with a $0.5 \AA$ probe and SASA with a $1.4 \AA$ probe (SASApack score $>0 \AA^{2}$ ) indicates poor packing and the presence of small voids that cannot be filled with water. By construction, $50 \%$ of proteins in the protein database have average per-residue SASApack scores $<0.0 \AA^{2}$. The SASAprob score is the probability of observing an amino acid in the PDB in a similar environment with a higher SASApack score. A SASAprob score of 0.95 indicates that a residue is more tightly packed than $95 \%$ of similar residues in the PDB.

Protein Expression and Purification. Genes for the redesigned proteins were constructed with cassette mutagenesis in the pET21b expression vector. The proteins were expressed in the E. coli BL21 strain at $37^{\circ} \mathrm{C}$ with $0.5 \mathrm{mM}$ isopropyl $\beta$-D-thiogalactoside used for 
induction. The proteins were purified with a $\mathrm{Ni}^{+}$affinity column followed by size-exclusion chromatography (Superdex 75).

NMR. The redesigned proteins $(\approx 1 \mathrm{mM})$ were equilibrated in a 20 $\mathrm{mM}$ sodium phosphate $/ 0.5 \mathrm{M} \mathrm{NaCl}(\mathrm{pH} 7.4)$ buffer, and onedimensional ${ }^{1} \mathrm{H}$ NMR spectra were recorded at $25^{\circ} \mathrm{C}$ on a $700-\mathrm{MHz}$ Varian (Palo Alto, CA) spectrometer.

Circular Dichroism. CD data were collected on a Jasco (Tokyo, Japan) J-810/815 CD spectrometer with a 1-mm path-length cuvette. Protein concentrations were $50 \mu \mathrm{M}$ in a solution of $10 \mathrm{mM}$ potassium phosphate, $\mathrm{pH}$ 7.0. The CD signal was monitored at 200 $\mathrm{nm}$ as a function of temperature $\left(10-90^{\circ} \mathrm{C}\right)$. The fraction of unfolded was calculated assuming that the $\mathrm{CD}$ signal of the unfolded and folded protein varies linearly with temperature.

Crystallization, X-Ray Diffraction, and Structure Determination. The hanging-drop vapor diffusion method was used for crystallization trials of the three designed proteins at room temperature. LoopA $(\approx 20 \mathrm{mg} / \mathrm{ml}$ in $100 \mathrm{mM} \mathrm{NaCl} / 20 \mathrm{mM}$ Tris ( $\mathrm{pH} 7.4)$ was mixed with an equal volume of well buffer of $2.0 \mathrm{M}$ ammonium sulfate, $\mathrm{pH}$ 3.0/10\% additive $0.1 \mathrm{M}$ cupric chloride (Hampton Research, Aliso Viejo, CA) was added to the drop. Ethylene glycol (20\%) was used

1. Hill RB, Raleigh DP, Lombardi A, DeGrado WF (2000) Acc Chem Res 33:745-754.

2. Harbury PB, Plecs JJ, Tidor B, Alber T, Kim PS (1998) Science 282:1462-1467.

3. Hecht MH, Richardson JS, Richardson DC, Ogden RC (1990) Science 249:884-891.

4. Hecht MH (1994) Proc Natl Acad Sci USA 91:8729-8730.

5. Kortemme T, Ramirez-Alvarado M, Serrano L (1998) Science 281:253-256.

6. Kuhlman B, Dantas G, Ireton GC, Varani G, Stoddard BL, Baker D (2003) Science 302:1364-1368.

7. Kuhlman B, O'Neill JW, Kim DE, Zhang KY, Baker D (2002) J Mol Biol 315:471-477.

8. Dahiyat BI, Mayo SL (1997) Science 278:82-87.

9. Regan L (1994) Curr Biol 4:656-658.

10. Minor DL, Jr, Kim PS (1994) Nature 367:660-663.

11. Munoz V, Serrano L (1995) Curr Opin Biotechnol 6:382-386.

12. Nagi AD, Regan L (1997) Fold Des 2:67-75.

13. Butterfoss GL, Kuhlman B (2006) Annu Rev Biophys Biomol Struct 35:49-65.

14. Yin H, Slusky JS, Berger BW, Walters RS, Vilaire G, Litvinov RI, Lear JD, Caputo GA, Bennett JS, DeGrado WF (2007) Science 315:1817-1822.

15. Looger LL, Dwyer MA, Smith JJ, Hellinga HW (2003) Nature 423:185-190.

16. Huang PS, Love JJ, Mayo SL (2005) J Comput Chem 26:1222-1232.

17. Desjarlais JR, Handel TM (1999) J Mol Biol 290:305-318.

18. Fu X, Apgar JR, Keating AE (2007) J Mol Biol 371:1099-1117.

19. Thanki N, Zeelen JP, Mathieu M, Jaenicke R, Abagyan RA, Wierenga RK, Schliebs W (1997) Protein Eng 10:159-167.

20. Ginalski K (2006) Curr Opin Struct Biol 16:172-177.

21. Rohl CA, Strauss CE, Misura KM, Baker D (2004) Methods Enzymol 383:66-93.

22. Rohl CA, Strauss CE, Chivian D, Baker D (2004) Proteins 55:656-677.

23. Gray JJ, Moughon S, Wang C, Schueler-Furman O, Kuhlman B, Rohl CA, Baker D (2003) J Mol Biol 331:281-299.

24. Schueler-Furman O, Wang C, Bradley P, Misura K, Baker D (2005) Science 310:638-642. as the cryoprotectant. Crystals of LoopB $(\approx 35 \mathrm{mg} / \mathrm{ml}$ in $100 \mathrm{mM}$ $\mathrm{NaCl} / 20 \mathrm{mM}$ Tris, $\mathrm{pH}$ 7.4) were grown against a well buffer of 3.8 $\mathrm{M}$ sodium formate/5\% glycerol, $\mathrm{pH} 7.5$. Glycerol (20\%) was used as the cryoprotectant. Diffraction data of LoopA were collected at the Advanced Photon Source at Argonne National Laboratory, Beamline 22-ID (Southeast Regional Collaborative Access Team). Diffraction data of LoopB were collected at the Beamline X29A at Brookhaven National Laboratory.

The data were indexed and processed with the program HKL2000 (41). The structures of both LoopA and LoopB were solved by molecular replacement, using the programs AmoRe (42) and Phaser $(43,44)$. WT tenascin (PDB code 1TEN) was used as the initial search model. The models were then refined against the synchrotron data to resolutions of 1.45 and $2.1 \AA$, respectively (SI Table 5). Alternating cycles of model building with the program $\mathrm{O}$ (45) and refinement with the programs CNS (46) and Refmac (47) were used to determine the final structure. The geometry of the final model was assessed with Procheck software (48).

We thank Howard Robinson and Jillian Orans for collection of diffraction data from beamline X29 at NSLS and 22-ID (Southeast Regional Collaborative Access Team) at the Advanced Photon Source at Argonne National Laboratory. This work was supported by an award from the W. M. Keck Foundation and National Institutes of Health Grant GM073960.

25. Kortemme T, Morozov AV, Baker D (2003) J Mol Biol 326:1239-1259.

26. Lazaridis T, Karplus M (1999) Proteins 35:133-152.

27. Leahy DJ, Hendrickson WA, Aukhil I, Erickson HP (1992) Science 258:987991.

28. Carr PA, Erickson HP, Palmer AG, III (1997) Structure (London) 5:949-959.

29. Batori V, Koide A, Koide S (2002) Protein Eng 15:1015-1020.

30. McDonald IK, Thornton JM (1994) J Mol Biol 238:777-793.

31. Sood VD, Baker D (2006) J Mol Biol 357:917-927.

32. Simons KT, Kooperberg C, Huang E, Baker D (1997) J Mol Biol 268:209-225.

33. Canutescu AA, Dunbrack RL, Jr (2003) Protein Sci 12:963-972.

34. Dunbrack RL, Jr, Cohen FE (1997) Protein Sci 6:1661-1681.

35. Bower MJ, Cohen FE, Dunbrack RL, Jr (1997) J Mol Biol 267:1268-1282.

36. Liu Y, Eisenberg D (2002) Protein Sci 11:1285-1299.

37. Hom GK, Lassila JK, Thomas LM, Mayo SL (2005) Protein Sci 14:1115-1119.

38. Kuhlman B, O’Neill JW, Kim DE, Zhang KY, Baker D (2001) Proc Natl Acad Sci USA 98:10687-10691.

39. Kuhlman B, Baker D (2000) Proc Natl Acad Sci USA 97:10383-10388.

40. Wang C, Bradley P, Baker D (2007) J Mol Biol 373:503-519.

41. Otwinowski Z, Minor W (1997) Methods Enzymol 276:307-326.

42. Navaza J (1994) Acta Crystallogr A 50:157-163.

43. McCoy AJ, Grosse-Kunstleve RW, Storoni LC, Read RJ (2005) Acta Crystal$\log$ D 61:458-464.

44. Storoni LC, McCoy AJ, Read RJ (2004) Acta Crystallogr D 60:432-438.

45. Jones TA, Zou JY, Cowan SW, Kjeldgaard M (1991) Acta Crystallogr A 47(2):110-119.

46. Brunger AT, Adams PD, Clore GM, DeLano WL, Gros P, Grosse-Kunstleve RW, Jiang JS, Kuszewski J, Nilges M, Pannu NS, et al. (1998) Acta Crystallogr D 54:905-921.

47. Murshudov GN, Vagin AA, Dodson EJ (1997) Acta Crystallogr D 53:240-255.

48. Laskowski RA, MacArthur MW, Moss DS, Thornton JM (1993) J Appl Cryst 26:283-291. 